\title{
Design and setting up of a system for remote monitoring and control on auxiliary machines in electric vehicles
}

\author{
Vasil Dimitrov ${ }^{1, *}$, and Petko Kostadinov ${ }^{1}$ \\ ${ }^{1}$ Todor Kableshkov University of Transport - Sofia, Department of Power Engineering and Electrical Equipment in Transport, \\ Faculty of Telecommunications and Electrical Equipment in Transport, 158 Geo Milev St., 1574 Sofia, Bulgaria
}

\begin{abstract}
Systems for remote monitoring and control of the proper operation, energy consumption, and efficiency of the controlled objects are very often used in different spheres of industry, in the electricity distribution network, etc. Various types of intelligent energy meters, PLCs and other control devices are involved in such systems. Proper operation of the auxiliary machines in electric vehicles is of great importance and implementation of a system for their remote monitoring and control is useful and ensures reliability and increased efficiency. A system has been designed and built using contemporary devices. An asynchronous motor is controlled by a soft starter and opportunities for remote monitoring (by an intelligent energy meter) and control (by a PLC and Touch panel) have been provided. Soft starters are widely used in industry for control on asynchronous drives when speed regulation is not a mandatory requirement. They are cheaper than inverters and frequency converters and allow for temporal reduction of the torque and current surge during start-up, as well as smooth deceleration. Therefore they can also be used in electric vehicles to control auxiliary machines (pumps, fans, air coolers, compressors, etc.). The present paper presents a methodology for their design and setting up.
\end{abstract}

\section{Introduction}

Proper operation of the auxiliary machines in electric vehicles is very important. They have to provide reliable cooling of the traction equipment, as well as compressed air with a certain pressure required for the systems controlling the doors, the brakes, and some pneumoelectrical devices. On the other hand, increased energy costs have resulted in greater emphasis on the power consumption of drive systems. It is extremely important to utilize the full potential for minimizing energy consumption using systems for control on motors with improved efficiency and performance. Different types of low-voltage asynchronous (induction) motors are very often used for driving auxiliary machines. These motors have minimized dimensions and weight, higher efficiency and enhanced reliability as opposed to DC motors.

In this paper, a laboratory system for control of an asynchronous motor has been designed and built with contemporary devices. Opportunities for remote monitoring and control have been provided. The developed methodology for its design and setting up can be used when building systems for control of auxiliary machines in different spheres of industry and transport.

\section{Selecting the motors}

Auxiliary machines in electric vehicles are mostly pumps, fans, air coolers, compressors. The driving motor has to be selected according to the rated power and speed of the machine [1]. The following correlation can be used: - calculation power $P_{\text {calc }}$ of the motor driving a pump:

$$
P_{\text {calc }}=\frac{k_{s} \cdot Q \cdot H \cdot \gamma}{\eta_{p} \cdot \eta_{t}}, W,
$$

where $k_{s}=1,1-1,3$ is a stocking factor taking into account the possibility of overloading;

$Q$ - pump flow rate, $\mathrm{m}^{3} / \mathrm{s}$;

$H$ - computational feed height (head, liquid column), m; $\gamma$ - volume weight of the liquid, $\mathrm{N} / \mathrm{m}^{3}$;

$\eta_{p}$ - efficiency of the pump;

$\eta_{t}-$ efficiency of the gear (transmission used).

- calculation power $P_{\text {calc }}$ of the motor driving a fan:

$$
P_{\text {calc }}=\frac{k_{s \cdot} \cdot Q \cdot p}{\eta_{f} \cdot \eta_{t}}, W
$$

where $k_{s}=1,25-2$ at motor power under $5 \mathrm{~kW}$ and $k_{s}=1,1-1,25$ at motor power above $5 \mathrm{~kW}$;

$Q$ - fan volume flow rate, $\mathrm{m}^{3} / \mathrm{s}$;

$p$ - computational fan pressure, $\mathrm{N} / \mathrm{m}^{2}$;

$\eta_{f}$ - efficiency of the fan.

- calculation power $P_{\text {calc }}$ of the motor driving a piston compressor:

$$
P_{\text {calc }}=\frac{Q \cdot A_{s}}{\eta_{c} \cdot \eta_{t}}, W,
$$

where $Q$ is the compressor performance, $\mathrm{m}^{3} / \mathrm{s}$; $A_{s}$ - specific work required to compress $1 \mathrm{~m}^{3}$ of air to the appropriate pressure, $\mathrm{J} / \mathrm{m}^{3}$ (determined in Table 1); $\eta_{c}$ - efficiency of the compressor.

*Corresponding author: vdimitroff@abv.bg 
Table 1. Determining the specific work $A_{s}$ required to compress $1 \mathrm{~m}^{3}$ of air to the appropriate pressure $p$.

\begin{tabular}{|c|c|}
\hline Pressure: $p, \mathrm{~Pa}$ & Specific work: $A_{s}, \mathrm{~J} / \mathrm{m}^{3}$ \\
\hline $1,5.10^{5}$ & $42.10^{3}$ \\
\hline $2.10^{5}$ & $73.10^{3}$ \\
\hline $3.10^{5}$ & $12.10^{4}$ \\
\hline $4.10^{5}$ & $16.10^{4}$ \\
\hline $5.10^{5}$ & $18.10^{4}$ \\
\hline $6.10^{5}$ & $21.10^{4}$ \\
\hline $8.10^{5}$ & $25.10^{4}$ \\
\hline $9.10^{5}$ & $26.10^{4}$ \\
\hline $12.10^{5}$ & $30.10^{4}$ \\
\hline
\end{tabular}

- calculation power $P_{\text {calc }}$ of the motor driving a rotary compressor:

$$
P_{\text {calc }}=\frac{168 \cdot Q \cdot\left(\mathrm{p}_{2}-\mathrm{p}_{1}\right)}{\eta_{c} \cdot \eta_{t}}, W,
$$

where $p_{1}$ and $p_{2}$ are the pressures of suction and exhausted air, $\mathrm{Pa}$.

If the rated motor speed corresponds to the machine speed, a gear will not be necessary - a coupler should be used. As can be seen from the above-mentioned interdependencies, the motor power varies widely, but in most cases a motor with low or middle power is used. This is why a $3 \mathrm{~kW}$ motor has been selected and included in the developed laboratory system. An electromagnetic brake (EMB) has been built and coupled to the motor. It makes it possible to change the load of the motor [2].

\section{Design of a control system}

Asynchronous motors in contemporary drive systems can be controlled mostly by frequency converters (inverters) or soft starters. The speed regulation of the auxiliary machines in most cases is not a mandatory requirement. These drives need to be started and stopped smoothly but their speed can be constant through the operating modes. Intelligent devices called "Soft starters" can be used in those cases. They are cheaper than inverters and frequency converters and allow for temporal reduction of the torque and current surge during start-up, as well as smooth deceleration. Altistart 01 soft start / soft stop units ATS01N2 developed by Schneider Electric Company has been selected $[3,4]$. This unit controls the motor power supply to limit the starting current and for deceleration using thyristor regulator. It also features an internal bypass relay switched on during steady state operation. This device is equipped with 3 potentiometers for setting the start and deceleration time and for adjusting the starting voltage threshold according to the motor load. Two logic inputs provide Run/Stop commands and a third logic input can be used for the Boost function $(+24 \mathrm{~V}$ positive logic). One logic output indicates the end of starting and one relay output indicates that the voltage is switched off at the end of the deceleration stage. A green LED indicates that the unit is powered and a yellow LED indicates that the motor is powered at nominal voltage. The appropriate model is selected according to the technical data of the asynchronous motor. Altistart ATS01N2**QN is available for nominal voltage of $380 \mathrm{~V}$.
The ATS01N209QN model for nominal current 9A is appropriate for motors with power of 3-4 kW [5].

The necessary protection should be ensured and an appropriate 3 poles thermal magnetic motor circuit breaker MCB GV2-ME14 has been selected (rated current $10 \mathrm{~A}$ and thermal protection adjustment range $6 . .10 \mathrm{~A}$ depending on the load) [6].

The developed scheme and a photo of the laboratory system are shown in Fig. 1. The possibility of reverse has been provided by two buttons $\mathrm{F}$ (forward) and R (reverse) and circuit breakers $\mathrm{K} 1$ and $\mathrm{K} 2$. A stop button $\mathrm{S}$ and the necessary locks have been also included. An opportunity for electrodynamic brake has been provided by the transformer Trf, rectifier, button $\mathrm{Br}$ and circuit breaker $\mathrm{K}$. A $220 \mathrm{~V} / 36 \mathrm{~V}$ transformer has been selected and the DC voltage is $32 \mathrm{~V}$ at current $1 \mathrm{~A}$ (acceptable for the rectifier diodes). Circuit breakers and buttons for operation in $220 \mathrm{~V}$ circuits have been selected.

The logical inputs and outputs of the soft starter have been connected to the PLC Simatic S7-200. TP177micro Touch Panel has been configured for Start / Stop and Boost commands that are transmitted through the PLC to the soft starter. Two-wire control has been realized - the run and stop commands are controlled by a switch and single logic input LI2 of the soft starter. State "1" controls the run process and state " 0 " controls the stop process. Another switch has been configured for the Voltage Boost function via a third logic input of the soft starter activating this input enables the function for supplying a starting overtorque capable of overcoming any mechanical friction. When the input is in state " 1 ", the function is active and the starter supplies the rated voltage to the motor for a limited period of time $(200 \mathrm{~ms})$ before starting. The digital inputs of the PLC are used for receiving information about the drive condition. A bar has been configured on the Touch Panel and shows the motor speed indicated by the logic output LO1 of the soft starter (the bypass relay has been switched on and the motor has reached nominal speed). An alarm has also been configured on the Touch Panel and indicates a fault: the relay contact R1A-R1C of the Fault relay (built into the soft starter) closes with the Run command and opens when the motor voltage is around 0 with a decelerated stop or stop immediately on a fault.

Measuring equipment with high accuracy has been used. The power monitoring device Sentron PAC3200 developed by Siemens provides an analysis of the electrical system's current consumption and power flows [7-9]. It has been connected directly to the voltage system. Three 50/5 A current transformers CT are used for measuring the current (the maximum current at starting is $50 \mathrm{~A})$. This device precisely and reliably detects the power values of electrical consumers and provides important measured values for assessing the system state and power quality. It has a range of useful monitoring, diagnostics and service functions, a two-tariff active and reactive energy counters (imported and exported energy), a universal counter, and a working hours counter for monitoring the running time of loads.

Four function keys can be used for a simple configuration and quicker selection of the desired display menu. The integral Ethernet interface is provided for easy 
integration into the remote monitoring system. In the laboratory system, it is used for communication between the device and the computer (PC). Modbus TCP Ethernet protocol is used $(10 \mathrm{Mbps})$ and the relevant software product Powerconfig V3.7 has been installed on the PC. Sentron PAC3200 transmits the measured values to the supervisory systems, where the data can be further processed for display and control (there is an opportunity for exporting it to Excel tables). All the relevant system parameters can be shown on the graphical display or the monitor through the communication interface and the Powerconfig software:
- Voltage (Phase-neutral - $\mathrm{U}_{1-\mathrm{n}} / \mathrm{U}_{2-\mathrm{n}} / \mathrm{U}_{3-\mathrm{n}}$ and Phasephase $\mathrm{U}_{1-2} / \mathrm{U}_{2-3} / \mathrm{U}_{3-1}$ );

- Currents (per phase $-\mathrm{I}_{1} / \mathrm{I}_{2} / \mathrm{I}_{3}$ );

- Active power (per phase $- \pm \mathrm{P}_{1} / \pm \mathrm{P}_{2} / \pm \mathrm{P}_{3}$ and total $\left.\pm \mathrm{P}_{\text {total }}\right)$

- Reactive power (per phase $- \pm \mathrm{Q}_{1} / \pm \mathrm{Q}_{2} / \pm \mathrm{Q}_{3}$ and total $\left.\pm Q_{\text {total }}\right)$;

- Apparent power (per phase $-\mathrm{S}_{1} / \mathrm{S}_{2} / \mathrm{S}_{3}$ and total $\mathrm{S}_{\text {total }}$ );

- Power factor (per phase - $\left|\mathrm{PF}_{1}\right| /\left|\mathrm{PF}_{2}\right| /\left|\mathrm{PF}_{3}\right|$ and total $\left.\left|\mathrm{PF}_{\text {total }}\right|\right)$;

- Frequency f;

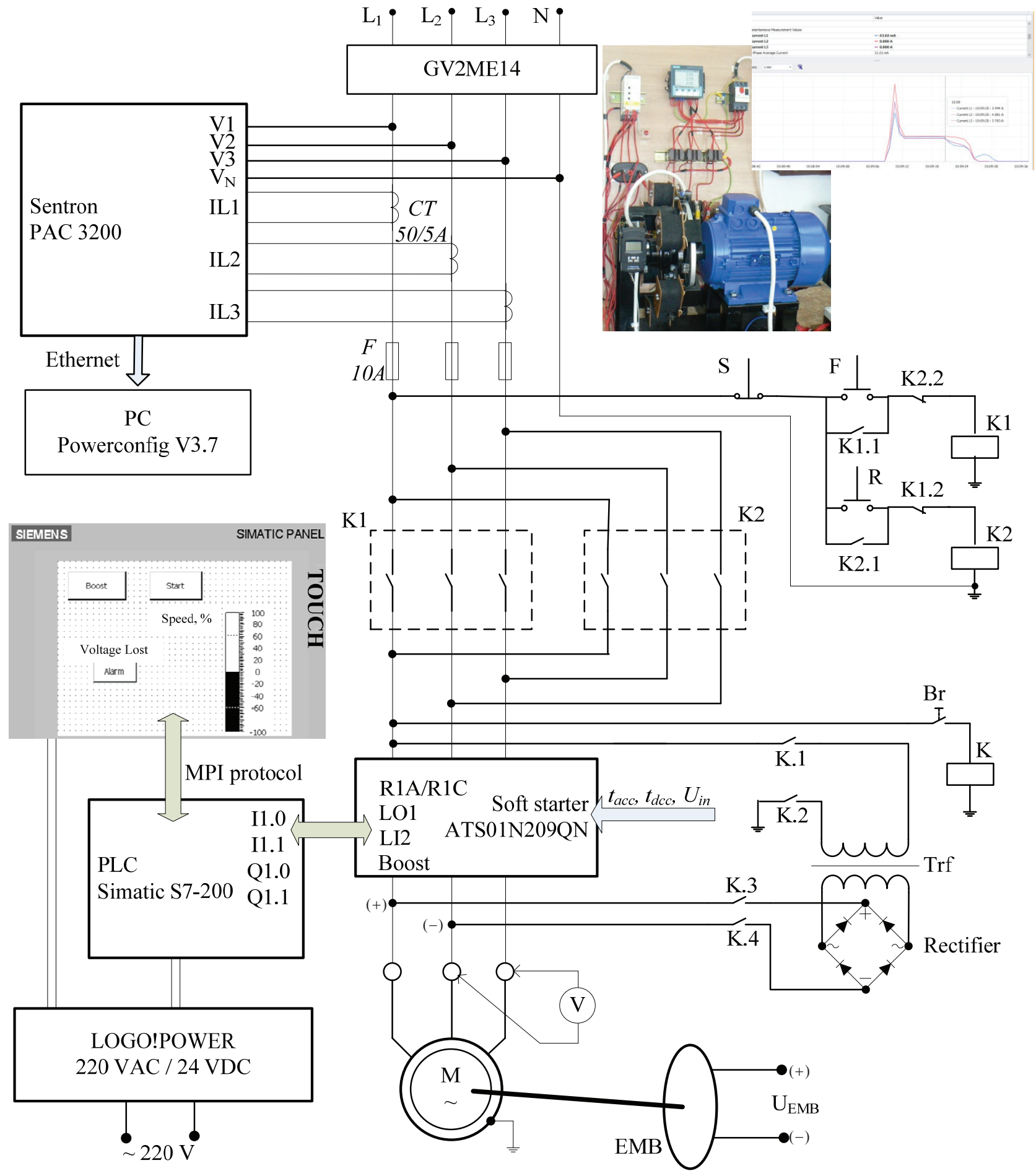

Fig. 1. Laboratory system - electrical scheme, exterior view of the drive and monitoring of its parameters on the PC. 
- Total harmonic distortion (THD) for voltage and current (per phase - THD-U ${ }_{1} /$ THD-U ${ }_{2} /$ THD-U ${ }_{3} /$ THD-I 1 THD-I I $_{2}$ THD-I $)_{3}$;

- Phase unbalance (Voltage);

- Min. / max. values (Voltage / Current / Power / Power factor / THD / Frequency / Three phase average voltage and current / Three phase average rating for active and reactive power), a possibility of reset is provided.

An example of remote monitoring of the drive performance on the PC (by the software Powerconfig) is shown in the Fig. 1. In addition, Sentron PAC3200 has a multifunctional digital input and output (simple logic functions for alarming can be set up).

Finally, it should be noted that most of the selected devices (or their analogs) may also be used in singlephase networks.

\section{Setting up the devices}

The presence of a PLC with a touch panel and a PC connected to the Sentron PAC3200 allows for simultaneous drive control, monitoring and parameters tracing and capturing. An appropriate setting up of devices to be used has to be made in advance.

Setting up the acceleration time $\left(t_{a c c}\right)$ using one of the soft starter potentiometers means that the time of the voltage ramp applied to the motor can be adjusted and gradual starting time can be achieved, which is dependent on the motor load. The initial voltage applied to the motor when switching on the soft starter $\left(U_{i n}\right)$ should also be set up in advance using the second potentiometer. It can be adjusted within the limits of $30-80 \% U_{n}$ according to the load. Therefore, the starting torque is $30 \ldots .80 \%$ of the one of a motor directly connected to the supply line.

The high values of the starting current and power can be reduced by increasing the acceleration time or decreasing the initial voltage. The correlation between the torque developed by the asynchronous motor and the supply voltage should be taken into account during the set up. The torque varies with the square of the voltage at a fixed frequency - for ex. at $U_{\text {in }}=1 / 2 U_{n}$ the starting torque will be reduced $1 / 4 M_{s t-n}$. Therefore, if the voltage is very low, the drive could not start at high load and some devices could be damaged. Then the Boost function must be activated. On the other hand, the gradual increase in the voltage prevents the instantaneous current peak on power-up.

Setting up the deceleration time $\left(t_{d c c}\right)$ can be done in a similar way using third potentiometer. A short time could lead to overvoltage that is not desirable. A smooth deceleration prevents the voltage drop on the line and damage of other devices in the network. This is why $t_{d c c}$ should also be adjusted according to the motor load.

Initial setup of the measuring device Sentron PAC 3200 includes specifying the operating parameters in the device settings. The connection type (3P4W -3 phases, 4 conductors, unbalanced load in the developed system), voltage (in our case direct measurement on the system) and measuring input voltage $(400 \mathrm{~V})$, current (primary 50 $\mathrm{A}$ and secondary $5 \mathrm{~A}$ ) must be properly set up. Parameterization of the language, regional settings, and password protection could also be made. The universal counter and the limit values could be defined.

The next step is setting up communication between Sentron PAC3200 and PC. The following parameters have to be defined in both devices: IP address, Subnet mask, Gateway address and Protocol used (Modbus TCP). The correct setting ensures that the two devices can work properly together.

The configuration of the parameters can be done either directly from the display with the function keys or through the communication interface using the PC.

\section{Conclusions}

The developed system ensures the proper operation of the controlled machines. The existence of a PLC with a touch panel and a PC connected to Sentron PAC3200 allows for simultaneous drive monitoring and control. Therefore, any failure can be discovered immediately and the reliability of the drive system is increased. The energy consumption can be optimized through the proper setup of the soft starter for each particular application. Many other drives can be also connected to the PLC and a common control system would be realized. The methodology for its design and set up can be used in developing systems for remote monitoring and control of different electrical drives.

\section{References}

1. G. Pavlov, V. Dimitrov, Design guide for electrical equipment (Propeller, Sofia, 2013)

2. V. Dimitrov, Development of laboratory system for electrical drives examinations, "MTC", 13 3/3, art. ID: 1246 (2015)

3. Soft starters Altistart 01 for asynchronous motors, Catalog (Schneider Electric, 2014)

4. Soft Starts for Single-Phase and Three-Phase Asynchronous Motors, Catalog (Schneider El., 2011)

5. ATS01N209QN Soft Starter for Asynchronous Motors, Product data sheet (Schneider El., 2011)

6. TeSys GV2-Circuit breaker - thermal - magnetic 6...10 A, Product data sheet (Schneider El., 2016)

7. E. Dimitrova, Conceptual description of the processes of remote control and monitoring of mobile objects, "MTC", 14 3/2, art. ID: 1394 (2016)

8. E. Dimitrova, Building automation and control systems, "MTC", 14 3/2, art. ID: 1395 (2016)

9. SENTRON PAC3200, Technical specification (Siemens, 2011)

10. http://w3.siemens.com/powerdistribution/global/en/lv /portfolio/pages/powerconfig.aspx 